\title{
Effect of gallium and arsenide adsorbed on graphene : a first-principles study on structural and electronic properties
}

\begin{abstract}
In this study, the adsorption influence of two different metals, gallium (Ga) and arsenide (As) adatoms on the stabilities and electronic structure of single graphene layer has been systematically studied using first-principles pseudopotentials calculations within the framework of density functional theory (DFT). The generalized gradient approximation used is PW91 exchange-correlation functional. The results of our calculations reveal that the adsorption of $\mathrm{Ga}$ atom on graphene resulted in electron transfer mainly from p-orbital of the $\mathrm{Ga}$ adatom to graphene and subsequently, altered the electronic state of graphene by shifting the Fermi level away from Dirac point, up to $\sim 1.5 \mathrm{eV}$. Meanwhile, the d-orbitals of Ga adatom have spin polarization at the Fermi level where the minority spin d-orbitals are unoccupied. The As adatom was found to have larger adsorption energy value on $\mathrm{H}, \mathrm{B}$ and $\mathrm{T}$ sites of graphene compared to $\mathrm{Ga}$ adatom. Thus, we described this energy difference as a result of the bonding configurations between both $\mathrm{Ga}$ and As atoms with carbon in the graphene structure. While B-site favored the adsorption of arsenic adatom, we found that the most favored adsorption site for $\mathrm{Ga}$ adatom on graphene is above $\mathrm{H}$-sites.
\end{abstract}

Keyword: Density functional theory; Graphene; Gallium and arsenic nanostructure. 